\title{
DEVELOPMENT OF 3D PIN-BY-PIN CORE SOLVER TORTIN AND COUPLING WITH THERMAL-HYDRAULICS
}

\author{
P. Mala, A. Pautz, H. Ferroukhi, A. Vasiliev \\ Paul Scherrer Institute \\ Forschungstrasse 111, 5232 Villigen, Switzerland
}

petra.mala@psi.ch, andreas.pautz@psi.ch,hakim.ferroukhi@psi.ch, alexander.vasiliev@psi.ch

\begin{abstract}
Currently, safety analyses mostly rely on codes which solve both the neutronics and the thermal-hydraulics with assembly-wise nodes resolution as multiphysics heterogeneous transport solvers are still too time and memory expensive. The pin-by-pin homogenized codes can be seen as a bridge between the heterogeneous codes and the traditional nodal assemblywise calculations. In this work, the pin-by-pin simplified transport solver Tortin has been coupled with a sub-channel code COBRA-TF. The verification of the $3 \mathrm{D}$ solver of Tortin is presented at first, showing very good agreement in terms of axial and radial power profile with the Monte Carlo code SERPENT for a small minicore and with the state-of-the-art nodal code SIMULATE5 for a quarter core without feedback. Then the results of Tortin+COBRA-TF are compared with SIMULATE5 for one assembly problem with feedback. The axial profiles of power and moderator temperature show good agreement, while the fuel temperature differ by up to $40 \mathrm{~K}$. This is caused mainly by different gap and fuel conductance parameters used in COBRA-TF and in SIMULATE5.
\end{abstract}

KEYWORDS: pin-by-pin, coupling, SP3, subchannel, fuel temperature

\section{INTRODUCTION}

The development of higher resolution multiphysics codes is currently a very active research field. However, these simulations are too computationally expensive for most industrial applications. Instead, the assemblywise nodal codes are commonly used nowadays. The thermal-hydraulic modules of nodal codes usually solve 1D (axial) two-phase flow equations with additional terms to take into account cross flow between assemblies [1]. While these codes can predict the local power by pin power reconstruction methods, the calculated moderator and fuel temperatures are only assembly-wise.

The pin-by-pin codes on the other hand can directly resolve the pin-wise power profile and, when coupled with a sub-channel thermal-hydraulic solver, they can directly predict the local safety parameters. This could be useful not only for transient calculations, but also for steady-state analysis of very heterogeneously loaded zones.

We have recently developed the pin-by-pin simplified transport solver Tortin and coupled it with a microscopic depletion code [2]. The eigenvalue and pin power results showed very good agreement with heterogeneous codes for 2D quarter core problems. In this work, the solver of Tortin was extended for three 
dimensions transport calculations and then coupled with the sub-channel thermal-hydraulic code COBRATF [3].

The results of Tortin are verified by comparison with the state-of-the-art nodal code SIMULATE5 [4]. Its neutronic solver is coupled with a thermal-hydraulic assembly-wise parallel channel module. The comparison of the codes is done firstly for a 3D quarter core with imposed temperatures to quantify the discrepancy between neutronics models, and then for one assembly with the thermal-hydraulic feedback. To further verify the 3D model of Tortin, a comparison with the Monte Carlo code SERPENT [5] is shown for a small minicore with heavy reflector.

\section{METHODOLOGY AND CODES DESCRIPTION}

Tortin is a pin-by-pin homogenized code, able to solve diffusion and SP3 equations. It employs the finite difference method for the spatial discretization. The code has been previously verified for 2D quarter core problems $[2,6]$ and the solver has been recently extended into three dimensions. All the results presented in the paper were obtained with the SP3 solver.

COBRA-TF (CTF) is a thermal-hydraulic code, solving the mass, energy, and momentum equations for three fields - liquid film, liquid droplets, and vapour. This nine conservation equations model can be spatially discretized into 3D Cartesian or sub-channel coordinates. In this work, the inlet temperature and mass flow rate and the outlet pressure were used as the boundary conditions. The fuel temperature can be calculated by solving the heat conductance equation. However, some important phenomena are not modelled, as for example the fuel pellet changes during burnup and its effect on the fuel conductance. The gap conductance calculated by INTERPIN [7] was used in this work. Since only one gap conductance value can be provided per each rod, the value corresponding to the maximum power of the rod was used.

The coupling scheme of the neutronics, thermal-hydraulics, and depletion solvers is depicted in Figure 1. Firstly, COBRA-TF is provided with axial power profile for each rod (84 axial layers were used in this work). The moderator density and the fuel temperature are calculated for each pin-cell (the moderator density is averaged over the 4 sub-channels adjacent to the given pin-cell, as shown in Figure 2). The pincell cross sections are interpolated for the given state parameters and they are passed into Tortin, which calculates the power distribution for COBRA-TF. Once the desired convergence on eigenvalue and the temperatures is reached, the calculation can continue with the next depletion step.

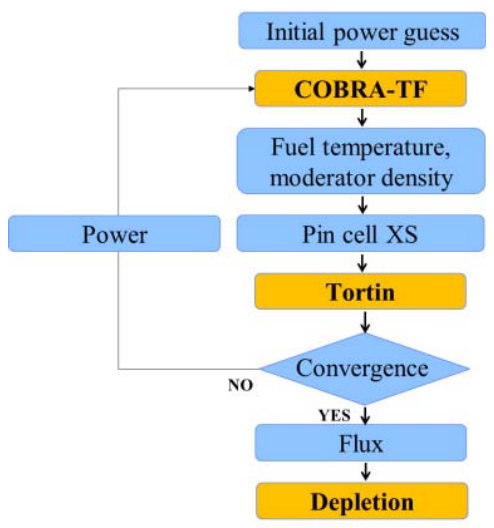

Figure 1. Calculation scheme of Tortin. 


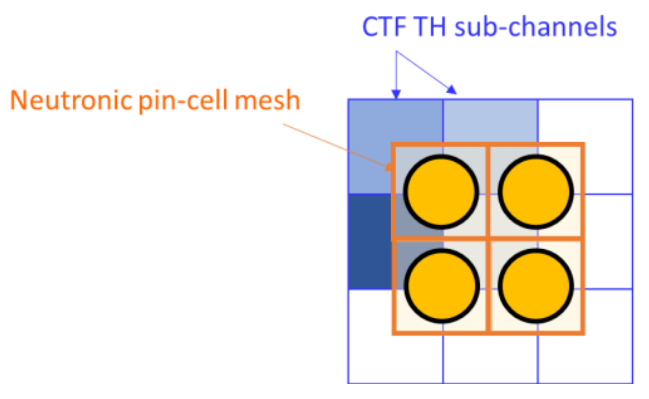

Figure 2. Principle of spatial coupling.

SIMULATE5 is a nodal code solving multi-group diffusion equation. It performs rehomogenization of the nodal cross sections based on flux obtained in 2D full core radial and 1D axial calculations with finer spatial resolution. The reflector cross sections are also rehomogenized. In this work, the 3D solver of SIMULATE5 was run with $2 \times 2$ nodes/assembly. The pin power is reconstructed by combining SIMULATE5 fluxes with pin power form functions obtained from CASMO5.

The thermal-hydraulic module of SIMULATE5 [8] models each assembly with an active coolant channel and a number of parallel water channels with the same submesh level as is used in the neutronics module. It can optionally take the cross-flow into account. The total mixture mass, steam mass, mixture enthalpy, and mixture momentum balance equations are solved for each axial node and the $3 \mathrm{D}$ fuel temperatures are obtained by solving the radial heat conduction equation for the average fuel pin of each node. It can also model the sub-cooled boiling, which can be important in large reactors such as EPR.

Apart from solving the 1D conduction equation, SIMULATE-5 can also calculate the fuel temperature by assuming its quadratic profile within the pin, with coefficients of the quadratic fit precomputed by INTERPIN. INTERPIN can predict the fuel-cladding performance dependence on burnup and power and can also provide the gap conductance values. The temperatures predicted by both the methods (solving the heat conduction equation and using the INTERPIN values) were compared in this work.

The cross sections for both Tortin and SIMULATE5 were generated with the lattice code CASMO-5 [9]. The 2D method of characteristics calculations were performed in 35 groups and with the P3 scattering source. Both the nodal and the pin-cell homogenized cross sections were produced in 8 energy groups. The pin-by-pin codes need to employ an equivalence technique between the heterogeneous lattice code and the pin-homogenized solutions, as the pin-cell homogenized cross sections calculated by a lattice code cannot preserve the reaction rates. The superhomogenization method [10] is used in Tortin.

For the verification of Tortin against SERPENT, the cross sections were produced with SERPENT to exclude any discrepancies coming from different nuclear libraries and the lattice codes methods. These cross sections were also produced in 8 energy groups.

\section{CASE DESCRIPTION}

The calculations were done for UK EPR fuel design [11]. The assemblies are uranium with $2.1-4.2 \%$ enrichment and containing up to 20 gadolinium-doped fuel rods. They have $17 \times 17$ rods and 24 guide tubes (Figure 3). The core is surrounded by heavy reflector, composed of stainless steel with water holes. The reflector is filled inside the steel core barrel. 

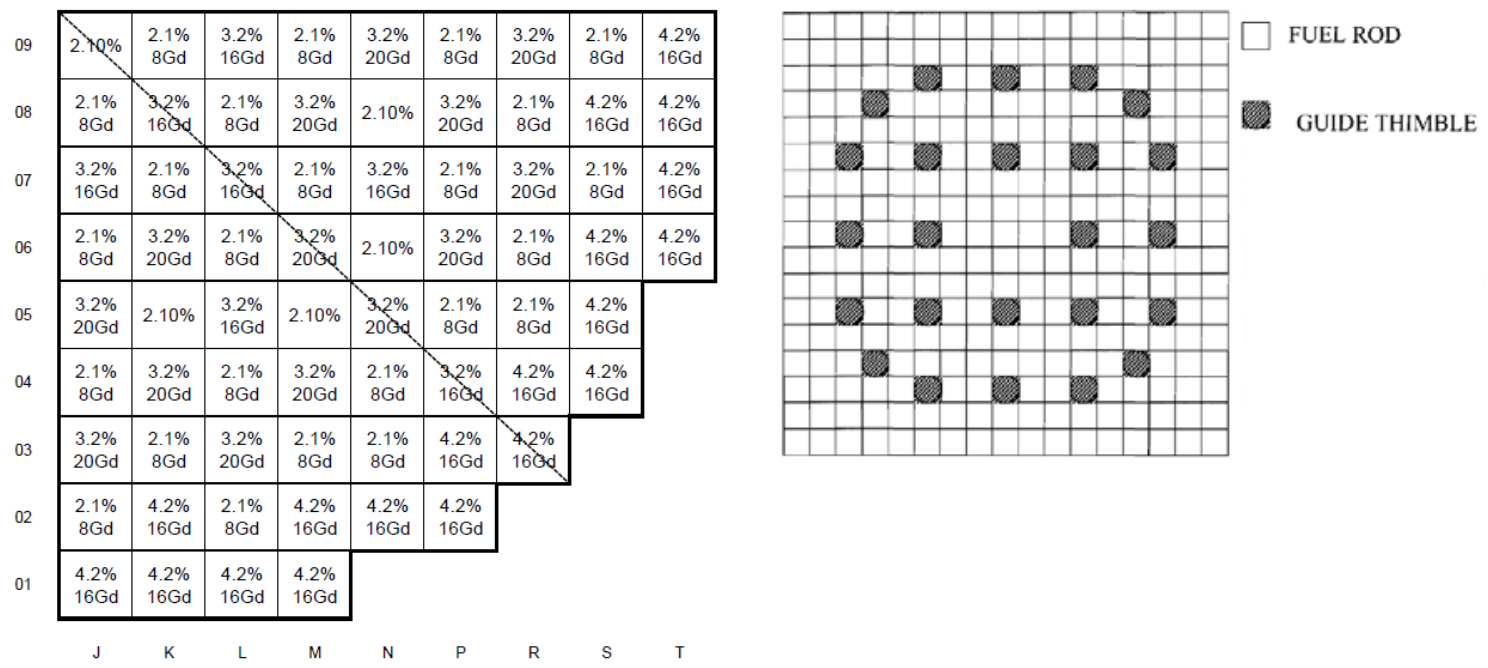

Figure 3. Core loading pattern for EPR first cycle and one assembly layout [11].

\section{RESULTS}

\subsection{D Quarter Core Problem without Feedback}

As a first verification of the 3D solver of Tortin, its results for the EPR quarter core were compared with SIMULATE5. This comparison was done for HZP with imposed thermal-hydraulic conditions in both codes, to allow quantification of the neutronics discrepancies separately from the other effects. The axial power profile of SIMULATE5 and the discrepancy of Tortin is shown in Figure 4 . There is about $0.8 \%$ difference in the nodes next to the reflectors, otherwise the power is very close. The axially integrated pin power discrepancy is shown in Figure 5 and is consistent with the previously published 2D results [2]. There is up to $3.5 \%$ discrepancy in the corner pins, caused probably by overprediction of the power by Tortin (similar behavior was observed when comparing Tortin against CASMO-5 2D results). Besides that, the discrepancy between the two codes is mostly within $2 \%$, the power profile of SIMULATE5 within the nodes tends to be flatter than that of Tortin. 


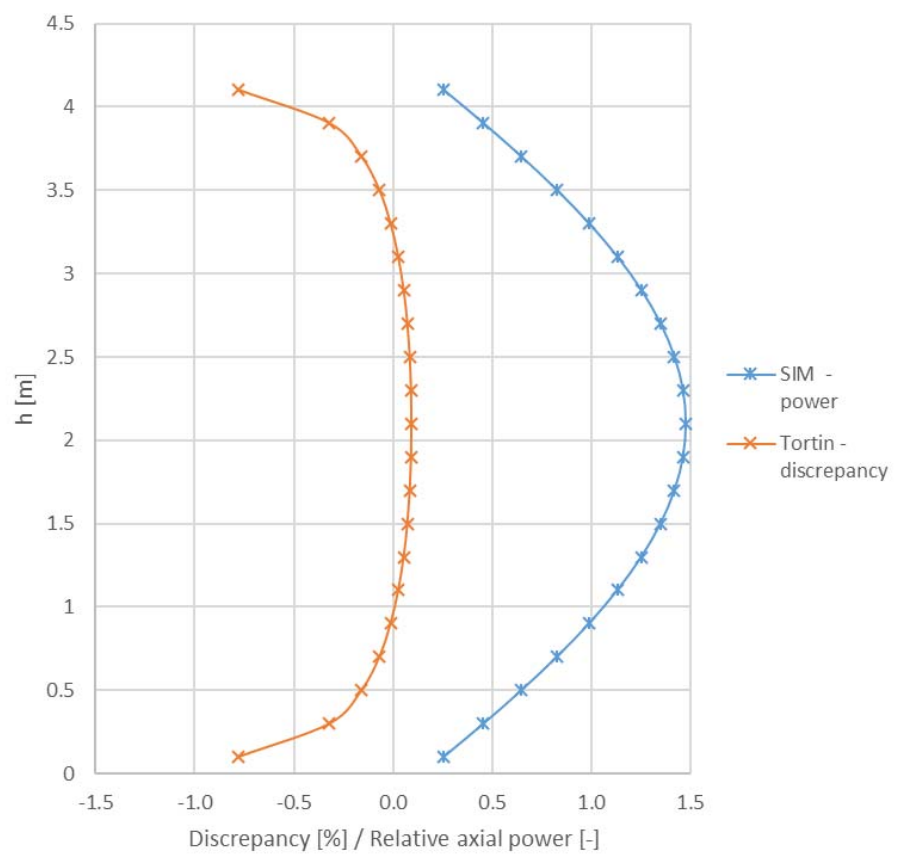

Figure 4. Axial power discrepancy for EPR core between Tortin and SIMULATE5.

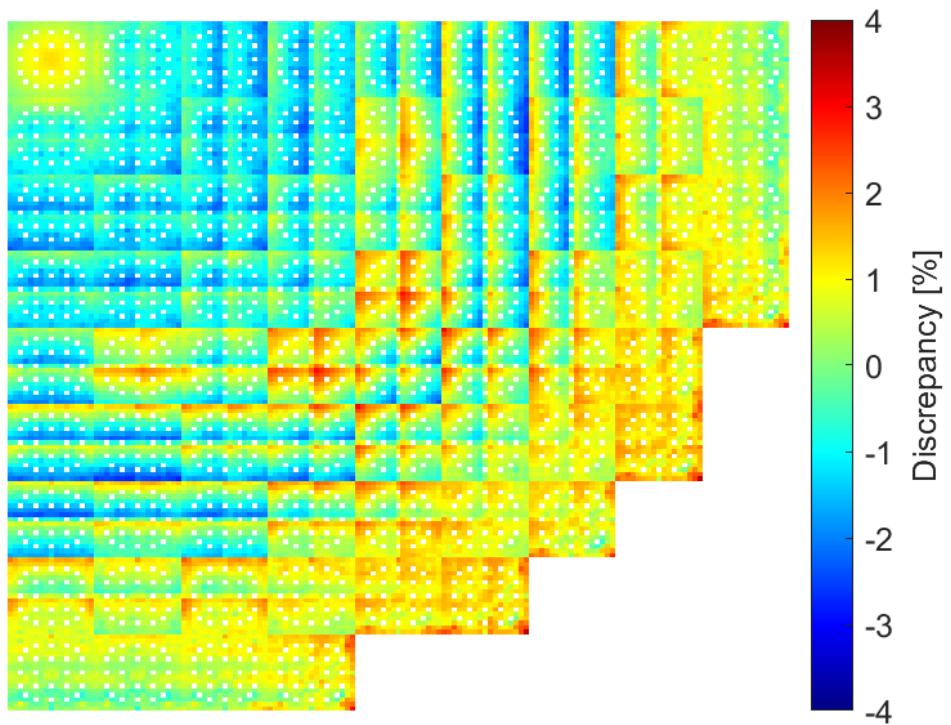

Figure 5. Radial pin power discrepancy for EPR core between Tortin and SIMULATE5.

\subsection{D Minicore without Feedback}

To further verify the accuracy of Tortin against a Monte Carlo solution, a small minicore calculation was done using SERPENT cross sections and the results were compared against SERPENT reference power distribution. The minicore is composed of 3 uranium assemblies in a row with a heavy reflector on the right side and with reflective boundary condition on the other sides. The axial reflectors consist of $5 \mathrm{~cm}$ of steel followed by $16 \mathrm{~cm}$ of water. The assemblies are $2 \mathrm{~m}$ high with 3 spacers of $4 \mathrm{~cm}$ each placed at 50,100 , and $150 \mathrm{~cm}$. The left assembly has control rods inserted into half of the assembly. 
The axial power profile as calculated by SERPENT and the errors of Tortin are shown in Figure 6 . The errors are very small, within $0.5 \%$ around the spacers and with maximum of about $1.5 \%$ around the axial reflectors. The errors of the axially averaged radial pin powers are shown in Figure 7 . They are also very small, with maximum of $1.3 \%$ in rods next to the radial reflector. The local power errors in one axial layer between 100 and $100.5 \mathrm{~cm}$ from the bottom, i.e. where the control rods and spacers are present, are shown in Figure 8. The statistical uncertainty of the reference values is below $0.4 \%$. The errors of Tortin around the reflector are below $2 \%$ and the power in the controlled assembly is underpredicted in average by $1.4 \%$.

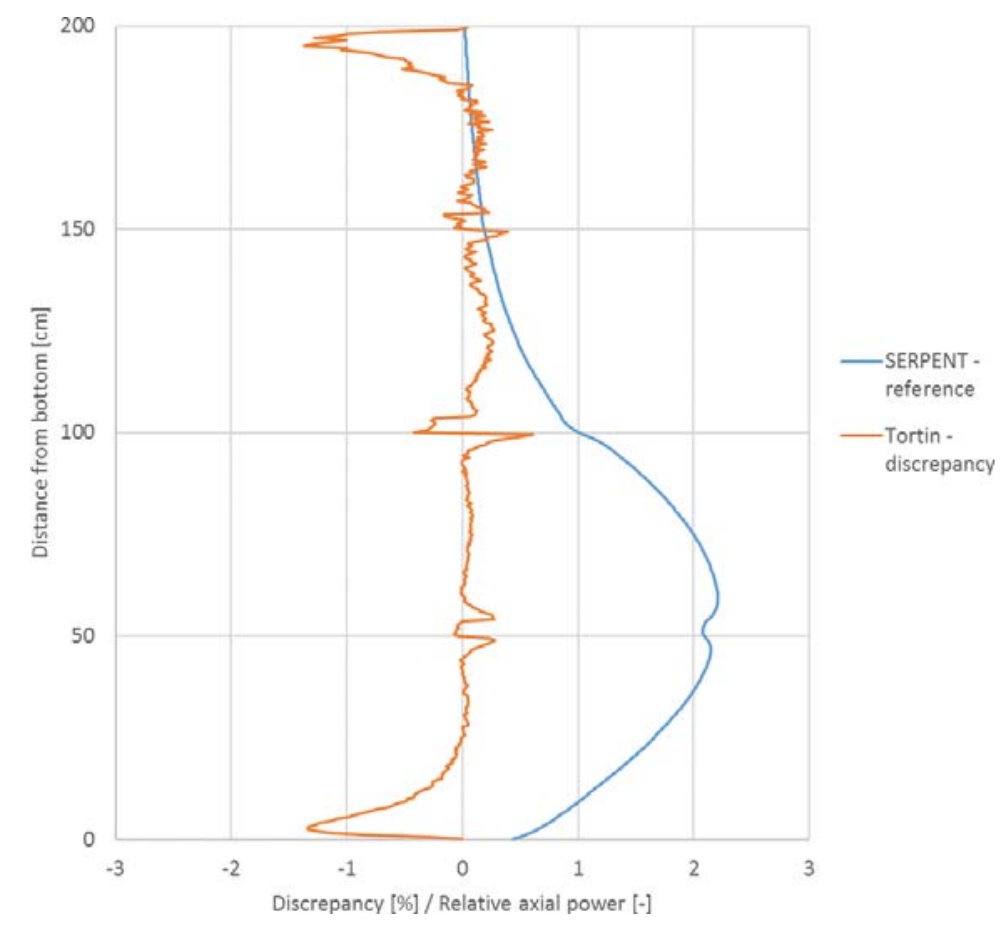

Figure 6. Axial power profile calculated by SERPENT and power error of Tortin using SERPENT cross sections.

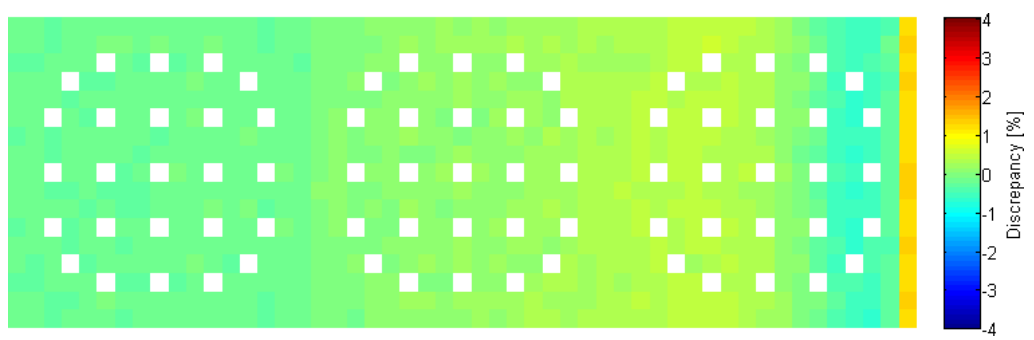

Figure 7. Axially averaged radial pin power error of Tortin using SERPENT cross sections and SERPENT reference values. 


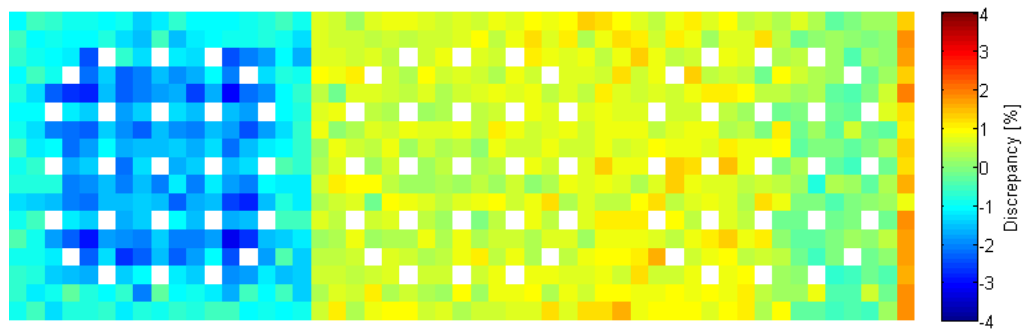

Figure 8. Pin power errors in axial layer between 100 and $100.5 \mathrm{~cm}$ from the bottom.

\subsection{One Assembly Problem with TH Feedback}

Tortin coupled with COBRA-TF (referred to as Tortin in the following text) was run for one assembly at HFP and the temperatures and the axial power profile were compared with SIMULATE5 results (Figure 9). The fuel temperature predicted by Tortin is higher than SIMULATE5 one by up to $40 \mathrm{~K}$. This is probably caused by differences in the gap conductance, the fuel conductance, and the fuel heat capacity. The moderator temperature of Tortin is slightly lower than that of SIMULATE5, by up to $0.8 \mathrm{~K}$. This could be caused by different enthalpy definition or differences in the mapping between the neutronics and TH in the two codes. Nevertheless, the discrepancy is small and should not have a big effect on the other results. The axial power profile shape of both codes is similar, with about $0.4 \%$ difference in the maximum relative power.

Since the uncertainties of the fuel and gap conductance and other related parameters are in general rather high [12], SIMULATE5 calculation was run also using the precomputed fuel temperature tables (provided by INTERPIN). The predicted maximum fuel temperature was $10 \mathrm{~K}$ lower, which caused about $0.3 \%$ difference in the maximum relative power.
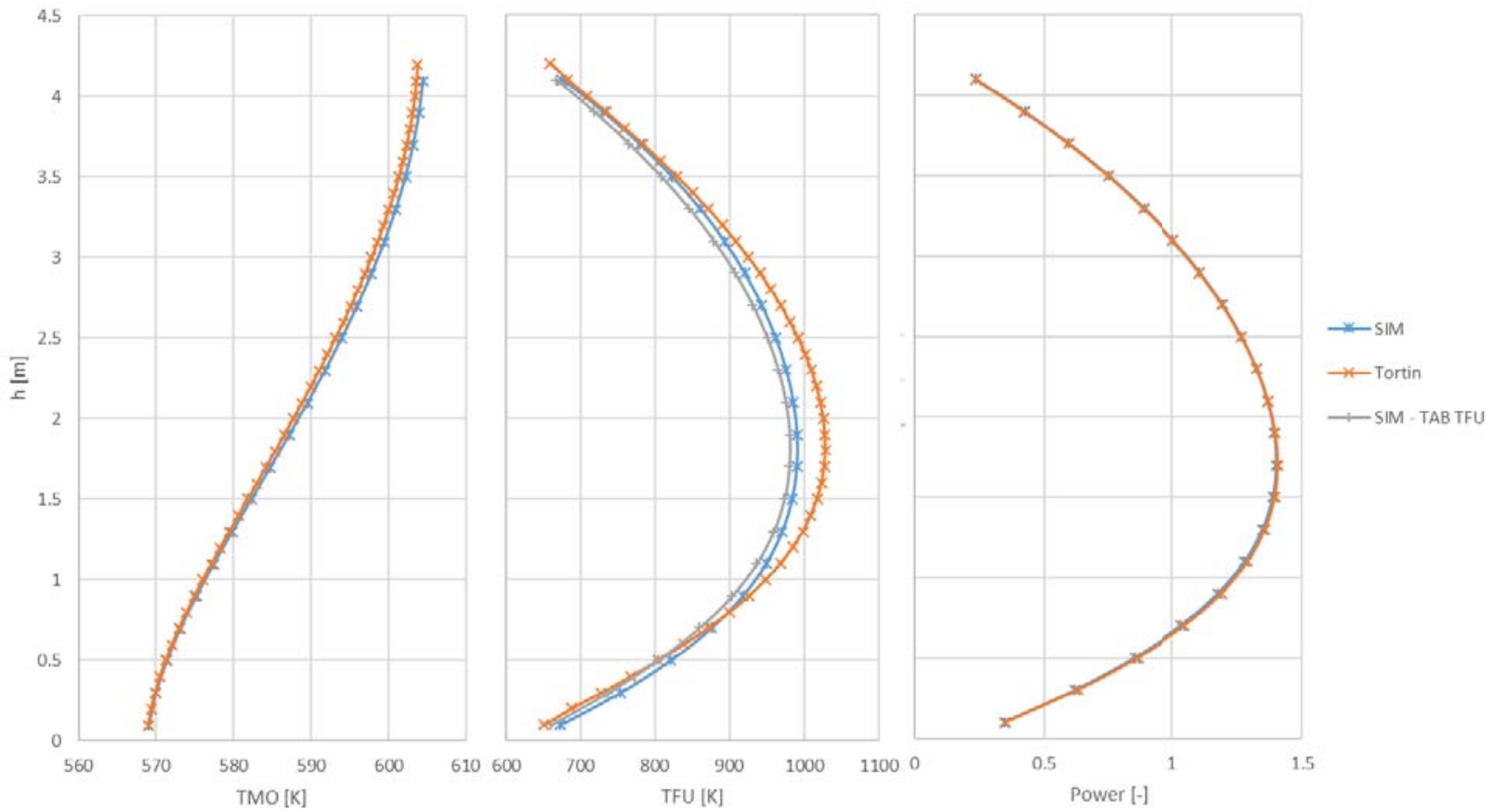

Figure 9. Moderator temperature, fuel temperature, and power - axial profile. 


\section{CONCLUSIONS}

The pin-by-pin homogenized simplified transport solver Tortin was extended into three-dimensions. The comparison with the Monte Carlo code SERPENT shows very good agreement, with around $0.5 \%$ error around the spacer grids and less than $1.5 \%$ error around the axial reflectors. The comparison with the SIMULATE5 nodal solver for the 3D quarter core shows also very good agreement, with about $0.8 \%$ differences in the power around the axial reflectors.

Tortin was then coupled with the sub-channel code COBRA-TF. The first comparison with SIMULATE5 shows good agreement in the moderator temperature and the axial power profile. However, there is up to $40 \mathrm{~K}$ difference in the fuel temperature. That is probably mainly caused by differences in the gap conductivity and the fuel model implemented in the two codes.

In the future, the fuel performance code Falcon could be used to provide COBRA-TF with these data. Once this is implemented, the testing of the code will be continued by full-core comparisons against SIMULATE5 and by validation with measurements.

\section{ACKNOWLEDGMENTS}

This work was partly funded by the Swiss Nuclear Safety Inspectorate ENSI (H-101230) and was conducted within the framework of the STARS program (http://www.psi.ch/stars).

\section{REFERENCES}

1. "The ARCADIA Reactor Analysis System for PWRs Methodology Description and Benchmarking Results," ANP-10297NP, Rev. 0 (2010).

2. P. Mala, A. Pautz, H. Ferroukhi, "EPR Fuel Cycle Depletion with Pin-by-pin Code Tortin and Nodal Code SIMULATE5," Proc. of M\&C 2019, Portland, USA, August 25-29 (2019).

3. R. K. Salko, M. N. Avramova, "CTF Theory Manual," NC State University (2018).

4. T. Bahadir, S. O. Lindahl, "Studsvik's Next Generation Nodal Code SIMULATE-5," Proc. of ANFM 2009, South Carolina, USA, April 12-15 (2009).

5. J. Leppänen, "Serpent - a Continuous-energy Monte Carlo Reactor Physics Burnup Calculation Code," VTT Technical Research Centre of Finland (2015).

6. P. Mala, "Pin-by-pin Treatment of LWR Cores with Cross section Equivalence Procedures and Higherorder Transport Methods," PhD thesis, EPFL (2018).

7. D. T. Hagrman, "INTERPIN-CS User's Manual," Studsvik Scandpower SOA-95/21 Rev.1 (2000).

8. S. O. Lindahl, T. Bahadir, G. Grandi, "SIMULATE5 - Methodogy," Studsvik Scandpower Report SSP10/465 (2011).

9. J. Rhodes, K. Smith, D. Lee, "CASMO-5 Development and Applications," Proc. of PHYSOR 2006, Vancouver, Canada, September 10-14 (2006).

10. A. Hebert, "A Consistent Technique for the Pin-by-Pin Homogenization of a Pressurized Water Reactor Assembly," Nuclear Science and Engineering, 113, pp. 227-238 (1993).

11. D. Page Blair, UK EPR Pre-construction Safety Report, UKEPR-0002-043 Issue 05 (2012).

12. M. Kruners, G. Grandi, M. Carlsson, "PWR Transient Xenon Modeling and Analysis using STUDSVIK CMS," Proc. of TopFuel 2010, Orlando, Florida, USA, September 26-29 (2010). 